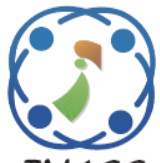

\title{
A Low-Complexity PAPR Reduction for Space-Time Block Code MIMO-OFDM by using modified-PTS with ABC-Concurrent Algorithm
}

\author{
Pitchaya Boontra $^{1} \quad$ Tanairat Mata $^{2 *} \quad$ Pisit Boonsrimuang $^{1}$ \\ ${ }^{I}$ Faculty of Engineering, King Mongkut's Institute of Technology Ladkrabang, Bangkok, 10520 Thailand \\ ${ }^{2}$ Faculty of Engineering, Rajamangala University of Technology Krungthep, Bangkok, 10120 Thailand \\ * Corresponding author's Email: tanairat.m@ mail.rmutk.ac.th
}

\begin{abstract}
One of the problems of using Orthogonal Frequency Division Multiplexing (OFDM) technique is the higher Peak-to-Average Power Ratio (PAPR) which leads to the fatal degradation of the system performance in the non-linear channel. To solve this problem, the partial transmit sequence (PTS) was proposed for Space-Time Block Code (STBC) with Multi-Input and Multi-Output (MIMO)-OFDM as STBC MIMO-OFDM system which can reduce the PAPR effectively. However, its complexity in the PAPR reduction process becomes higher in proportion to the increase of its considered cluster number. To solve this problem, this paper proposes a low-complexity PAPR reduction by using the modified-PTS with artificial bee colony $(\mathrm{ABC})$ and concurrent algorithm as ABC-Concurrent algorithm for STBC MIMO-OFDM system which can improve better PAPR with low-complexity and can perform better Bit-Error-Rate (BER) in the non-linear channel. The silent features of the proposed modified-PTS with ABCConcurrent algorithm are to reduce the PAPR with decreasing side information (SI) to half by modifying PTS with the concurrent algorithm and to reduce the complexity in PAPR reduction process by applying ABC algorithm for STBC MIMO-OFDM system. From the various results by using computer simulation, it can be confirmed that the proposed modified-PTS with ABC-Concurrent algorithm can reduce PAPR by approximately 3.4 and $0.4 \mathrm{~dB}$ at CCDF $10^{-3}$ as comparing with the original STBC MIMO-OFDM and conventional PTS methods respectively, and can use the complexity approximately only $19 \%$ in PAPR reduction process which is decreased a lot from using the conventional PTS method. Moreover, the proposed method can perform the better BER in the non-linear channel than the original STBC MIMO-OFDM and the conventional. PTS methods which is close to that of operating in nonlinear channel.
\end{abstract}

Keywords: STBC, MIMO, OFDM, Concurrent, PAPR.

\section{Introduction}

Up to today, MIMO-ODFM technique $[1,2]$ have been a lot of attention in many researches for the wireless communication because of the potential capability to high-speed data transmission, resistant to multipath fading channel, efficient in operating of spectrum etc. Also, the Space-Time Block Coded (STBC) [4, 5] which is one of the diversity techniques is most attractive for these purposes because the higher diversity gain can be gotten at the receiver from STBC signals which are sent from the transmitter with the multiple antennas. However, because of the higher PAPR which occurs in time domain OFDM signal, its performance would be degraded a lot especially in the non-linear channel such as the fatal degradation of BER, the larger nonlinear distortion at the output signal of the non-linear amplifier and spectrum frequency expansion.

To solve the high PAPR problem, many PAPR reduction methods have been proposed for OFDM signal such as data coding technique [5, 6], clipping and filtering technique $[7,8]$, phase scrambling technique i.e. selective mapping (SLM) [9, 10], PTS $[11,12]$. The PTS is one of the promising phase scrambling techniques for reducing the PAPR in OFDM signal which can reduce PAPR efficiently. In the main idea of the PTS technique, the whole subcarriers in each OFDM symbol are clustered in 


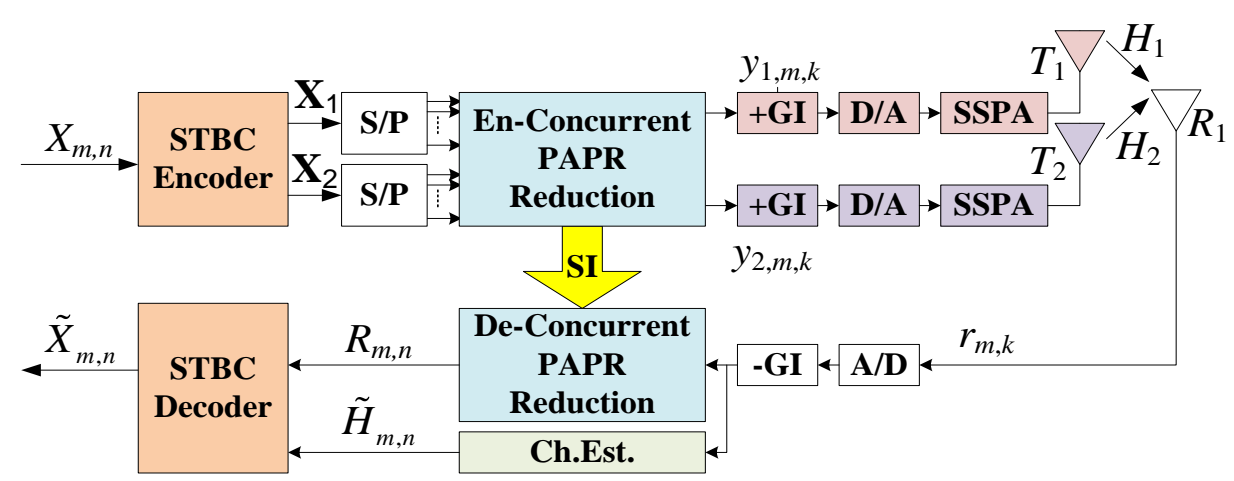

Figure. 1 The $2 \times 1$ STBC MIMO-OFDM systems with concurrent PAPR reduction

the frequency domain then multiplied by predetermined coefficient phases in the time domain after transformed by IFFT (Inverse Fast Fourier Transform) process, this process continues until the optimum PAPR value which provides the lower PAPR value can be selected for the transmitted signal. Phase information as $S I$ is required to inform to the receiver for correcting data demodulation in the PTS process. Although the high PAPR value could be reduced by using the PTS with increasing the number of clusters and pre-determined coefficient phases, its complexity in PAPR reduction process is increasing proportionally.

To reduce the high PAPR with low complexity which is the problem in PTS method as mentioned before, this paper focuses on the improvement of PAPR with low computational complexity. Therefore, this paper proposes the low-complexity PAPR reduction by using the modified-PTS with ABC-Concurrent algorithm for STBC MIMOOFDM systems. The silent features of the proposed method are to reduce PAPR by halving cluster of each OFDM symbol with multiplying two considered phases in each part separately as modified-PTS method and to reduce the computational complexity in the PAPR optimization process by modifying the artificial bee colony (ABC) algorithm to generate the pre-determined coefficient phases for the proposed PAPR reduction process. However, in STBC MIMO-OFDM system the PAPR reduction will employ in every transmitted antenna. From this fact, the SI-size required to inform to the receiver becomes larger which is proportional to the number of transmitted antennas. To solve this problem, the concurrent algorithm is applied for the proposed modified-PTS which can improve the better PAPR with decreasing the size of SI for STBC MIMO-OFDM system.

This paper is organized as follows. Section 2 presents the STBC MIMO-OFDM with PAPR reduction. Section 3 presents the modified-PTS with ABC-Concurrent algorithm. The effectiveness of the proposed PAPR reduction method can be verified from the various computer simulation results as compared with the other methods presented in Sect.4. Finally, we draw some conclusions in Sect.5.

\section{System model}

In this paper, we consider a MIMO-OFDM system which encodes by STBC [4] as STBC MIMO-OFDM. Fig. 1 shows the block diagram of STBC MIMO-OFDM system including two transmitted antennas $\left(T_{1}\right.$ and $\left.T_{2}\right)$ and one received antenna $\left(R_{1}\right)$ with the concurrent PAPR reduction technique. In the figure, the modulation data $\mathbf{X}_{\bmod }=$ $\left[X_{0, n}, X_{1, n}, \ldots, X_{L s y m-2, n}, X_{L s y m-1, n}\right](0 \leq n \leq M-1)$ with $M$ active data subcarriers will be encoded by STBC.

The encoded data symbols $\mathbf{X}_{1}$ and $\mathbf{X}_{2}$ for two transmitted antennas can be given by

$$
\begin{aligned}
& \mathbf{X}_{1}=\left[X_{0, n},-X_{1, n}^{*}, \cdots, X_{L_{s y m}-2, n},-X_{L_{s s m}-1, n}^{*}\right], \\
& \mathbf{X}_{2}=\left[X_{1, n}, X_{0, n}^{*}, \cdots, X_{L_{s y m}-1, n}, X_{L_{s y m}-2, n}^{*}\right],
\end{aligned}
$$

where $L_{s y m}$ is the number of OFDM symbols in one OFDM frame. The conjugation operator is denoted by *. From Eq.(1), $\mathbf{X}_{1}$ and $\mathbf{X}_{2}$ in the frequency domain are transformed into the time domain by IFFT process with $N$ points for every symbol then reduced their PAPR values explained in Sect.3. Finally, the time domain signal with optimum PAPR after adding the guard interval (GI) for avoiding the inter-symbol interference (ISI) in the multipath fading channel of each antenna is inputted to D/A converter and the non-linear amplifier respectively.

Meanwhile, the transmitted signal $s_{m}$ at $m$-th symbol after amplified by Input amplitude and Output Amplitude (AM/AM) characteristic for Solid State Power Amplifier (SSPA) [13] can be expressed as

$$
S_{m}=F\left[\left|y_{m}\right|\right] e^{j\left\{\arg \left(y_{m}\right)\right\}},
$$


where $y_{m}$ is the time domain signal after PAPR reduction and conversion characteristic of amplifier is denoted by $F[$.$] .$

The PAPR value of the transmitted time domain OFDM signal is defined by dividing the maximum signal power by the average signal power which can be defined by

$$
\operatorname{PAPR}\left\{y_{m}\right\}=\frac{\operatorname{Max}\left(y_{m}\right)}{\operatorname{Mean}\left(y_{m}\right)} .
$$

Furthermore, the complementary cumulative distribution function (CCDF) is evaluated as the PAPR for the PAPR reduction methods which can be defined by

$$
C C D F_{P A P R_{0}}=\text { Prob. }\left(P A P R>P A P R_{0}\right) \text {. }
$$

where $P A P R_{0}$ is the PAPR reference.

\section{Proposal of PAPR reduction by using the modified-PTS with ABC-Concurrent algorithm for STBC MIMO-OFDM}

\subsection{PAPR reduction by proposed modified-PTS method}

Fig. 2 shows the PAPR reduction by using PTS for OFDM signal. In the Fig.2, the modulation data $X_{m}$ at each $m$-th symbol will be divided into $V$ clusters $(1 \leq v \leq V)$ as $X_{m}^{v}$ then transformed by IFFT with $N$-points into time domain signal as $x_{m}^{v}$ which can be expressed by

$$
\operatorname{IFFT}\left\{X_{m, n}^{v}\right\}=x_{m, k}^{v}=\frac{1}{\sqrt{N}} \sum_{n=0}^{N-1} X_{m, n}^{v} \cdot e^{j \frac{2 \pi n k}{N}} .
$$

where the time index in IFFT is denoted by $k$. To find the optimum PAPR value for OFDM signal, $x_{m}^{v}$ is multiplied by weighting factor $b_{m}^{v}\left(=e^{j \phi_{m}^{v}}\right)$ where $\emptyset_{m}^{v} \in\left\{\frac{2 \pi l}{W} \mid l=0, \ldots, W-1\right\}$ and $W$ is the number of predetermined coefficient phases which can be given by

$$
y_{m}=\sum_{v=1}^{V} x_{m}^{v} \cdot b_{m}^{v}
$$

From Eq.(6), the optimum PAPR values are selected by the following equation

$$
\tilde{V}=\min _{W}\left[\max _{N}\left\{\left.y_{m}\right|_{\text {in Eq.(6) }}\right\}\right],
$$

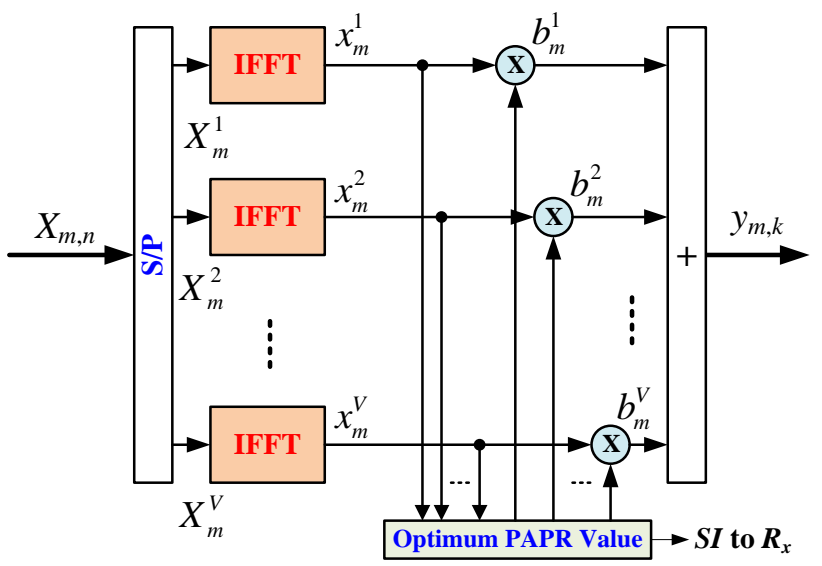

Figure.2 PAPR reduction by using PTS for OFDM signal

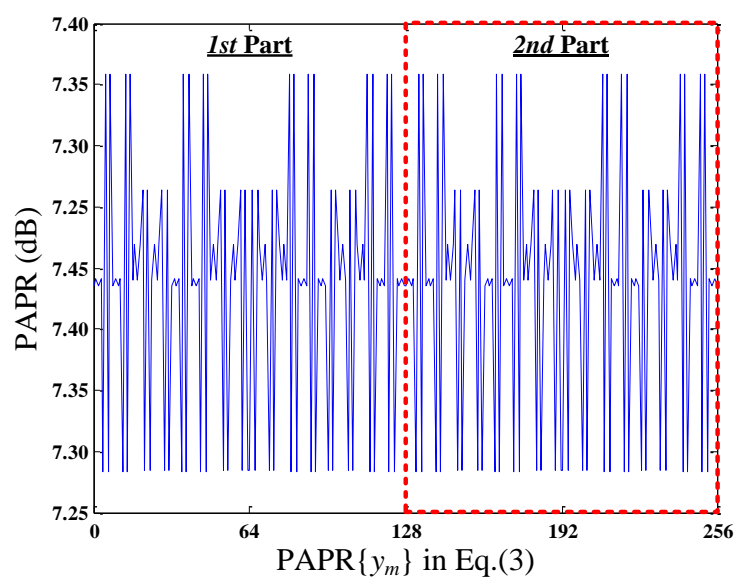

Figure.3 PAPR of OFDM signal after reducing by PTS in time domain

where $\tilde{V}$ is the desired weighting factor which gives the optimum PAPR values for the transmitted OFDM signal in the time domain.

From the Fig.2, if four data inputs are operated a QPSK modulation with four subcarriers as $1+i,-1+i$, $-1-i$ and $1-i$ respectively. In its PAPR reduction by using the PTS with two rotated phases $[0, \pi](W=2)$, the total possible PAPR values for the OFDM signal calculated in ref.[14] are 256. From Fig.3 which shows the possible 256 values of PAPR, it can be seen that the PAPR from 1th to 128th subcarriers have the same values as that from 129th to the last at 256th subcarriers. From this result, it can be divided the PAPR values into two parts as the $1 s t$ and $2 n d$ parts respectively, this means that the considered PAPR values can be computed only in the $1 s t$ part. From this reason, this paper proposes a modifiedPTS method to reduce the PAPR for OFDM signal which is shown in the Fig.4.

Fig. 4 shows the PAPR reduction by using the proposed modified-PTS for OFDM signal. In the proposed method, the whole data subcarriers in each 


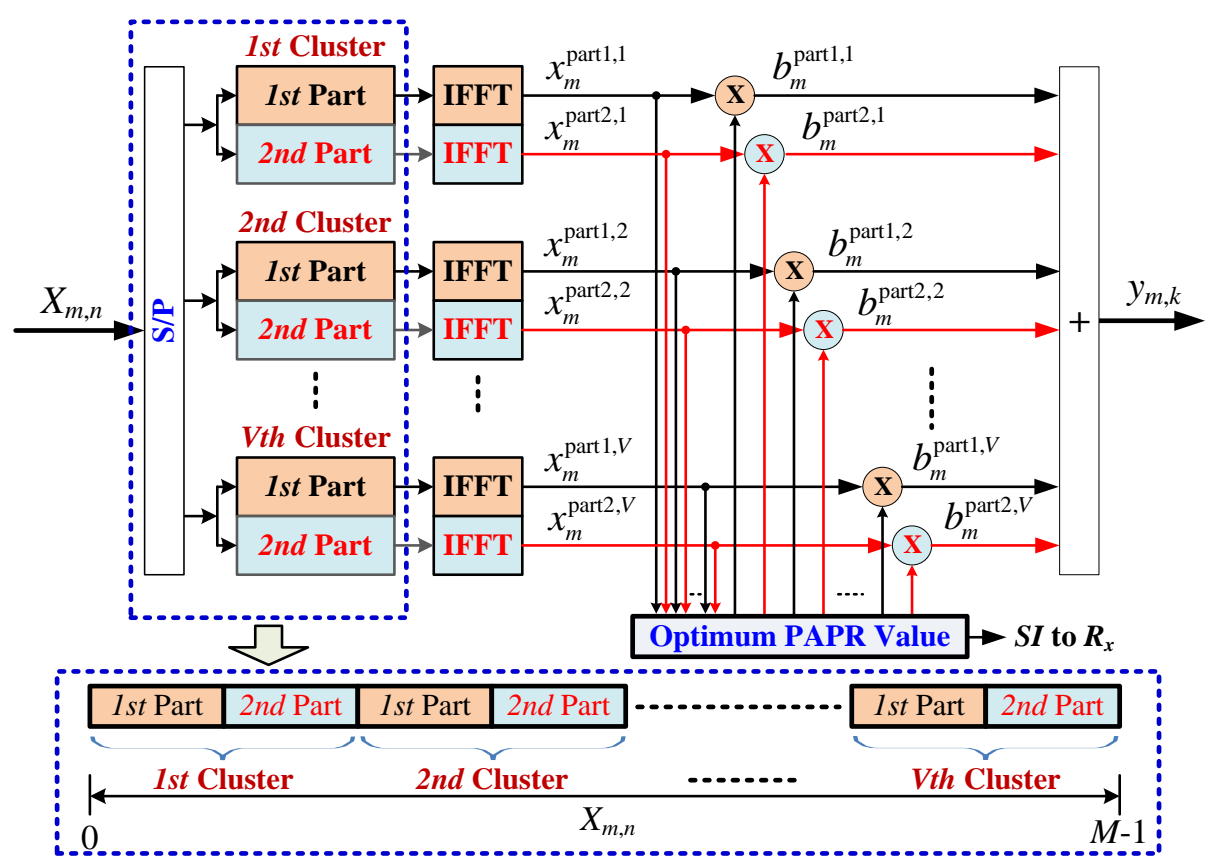

Figure.4 PAPR reduction by using proposed modified-PTS for OFDM signal

cluster are halved into two parts. The data subcarrier in the $1 s t$ and $2 n d$ parts of cluster are multiplied independently by two different pre-determined coefficient phases which can be given by

$$
y_{m}=\sum_{v=1}^{V}\left\{x_{m}^{\mathrm{part} 1, v} \cdot b_{m}^{\mathrm{part} 1, v}+x_{m}^{\mathrm{part} 2, v} \cdot b_{m}^{\mathrm{par} 2, v}\right\},
$$

where $b_{m}^{\text {part } 1, v}\left(=e^{j \phi_{m}^{\text {part } 1, v}}\right)$ and $b_{m}^{\text {part2,v}}\left(=e^{j \phi_{m}^{\text {part } 2, v}}\right)$ are the weighting factors multiplied in the $1 s t$ and 2nd parts respectively. The relationship between $\emptyset_{m}^{\text {part } 1, v}$ and $\emptyset_{m}^{\text {part } 2, v}$ can be defined by

$$
\phi_{m}^{\mathrm{part} 2, v}=\lambda \cdot \phi_{m}^{\mathrm{part} 1, v}
$$

where the constant value $\lambda$ is decided for the proposed modified-PTS to optimize the desired PAPR value in Sect.4 [15].

From Eqs.(8) and (9), it can be expected that the high PAPR of OFDM signal can be reduced by using the proposed modified-PTS with keeping the same SI-size as that of using the conventional PTS method.

\subsection{Proposed low-complexity PAPR reduction with modified ABC-phase optimization}

For reducing the complexity in the PAPR reduction process, the artificial bee colony $(\mathrm{ABC})$ algorithm [16] is applied for optimizing phases in the PAPR reduction procedure. In the basic concept of $\mathrm{ABC}$, the food sources optimization can be found by employed, onlooker and scout bees respectively which the 1st position of food source would be randomly generated. From its concept, the food source is defined as the optimum pre-determined coefficient phases for PAPR reduction including the employed or onlooker bees. A new food source is looked by employed bees from the neighbourhood of the previous food source. When the honey of the new food source becomes greater than that of the previous food source, the new food source is stored as the possible optimum solutions. From this process, it can modify the $\mathrm{ABC}$ for the solution of predetermined coefficient phases in the proposed modified-PTS which can be expressed as

$$
\hat{\phi}_{m, p, q}^{\mathrm{part} 1, v}=\hat{\phi}_{m, p, q}^{\mathrm{part} 1, v}+\beta_{p, q}\left\{\hat{\phi}_{m, p, q}^{\mathrm{part} 1, v}-\hat{\phi}_{m, s, q}^{\mathrm{part} 1, v}\right\},
$$

where $p$ and $s=1,2, \ldots, S$, and $q$ is $1,2, \ldots, V$ when $p \neq q$. The size of a randomly distributed the initial population and a random number between $[1,1]$ are represented by $S$ and $\beta_{p, q}$ respectively. From Eq.(10), the phase coefficients $\emptyset_{m}^{\text {part1,v }}$ of the $1 s t$ part in Eq.(9) when $W=4$ can be selected by the following equation

$$
\phi_{m}^{\mathrm{part} 1, v}=\left\{\begin{array}{ccc}
\frac{\pi}{2}, & \text { if } & \frac{\pi}{4} \leq \hat{\phi}_{m, p, q}^{\mathrm{part} 1, v}<\frac{3 \pi}{4} \\
\pi, & \text { if } & \frac{3 \pi}{4} \leq \hat{\phi}_{m, p, q}^{\mathrm{part} 1, v}<\frac{5 \pi}{4} \\
\frac{3 \pi}{2}, & \text { if } & \frac{5 \pi}{4} \leq \hat{\phi}_{m, p, q}^{\mathrm{part} 1, v}<\frac{7 \pi}{4} \\
0, & \text { if } & \text { else }
\end{array} .\right.
$$




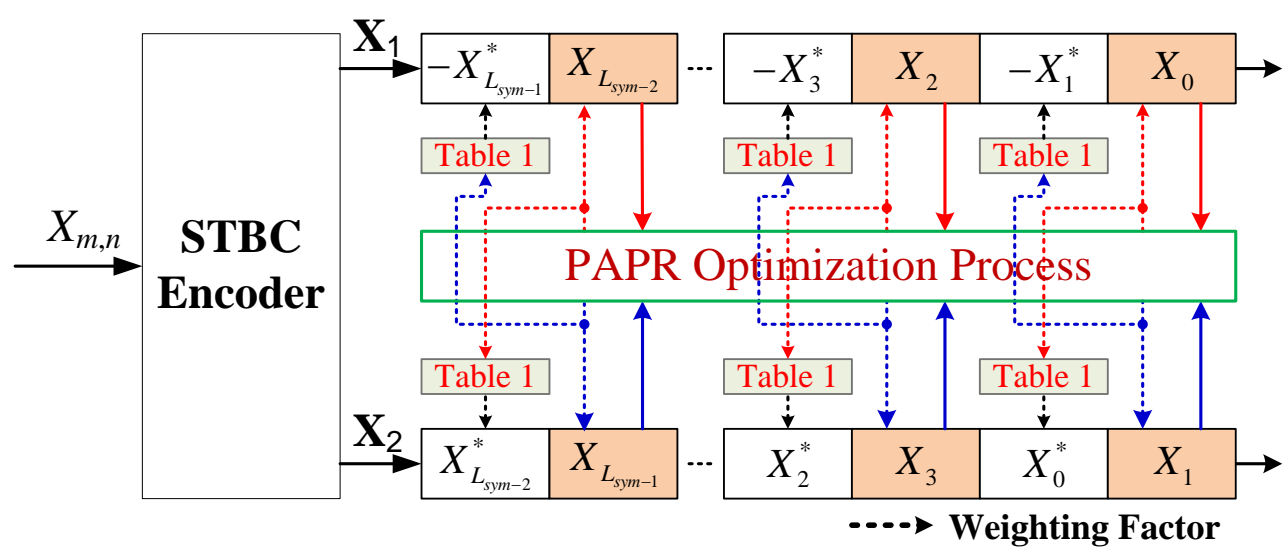

Figure.5 PAPR reduction with concurrent algorithm

\subsection{PAPR reduction by proposed modified-PTS with concurrent algorithm for STBC MIMO-OFDM system}

In STBC MIMO-OFDM system, more than one antenna is employed at the transmitter for sending the information data to the receiver. From this fact, the required SI-size which is informed to the receiver becomes larger which is proportional to the number of transmitted antennas. To solve this problem, the concurrent algorithm is applied for the proposed modified-PTS which can reduce PAPR with decreasing the SI-size to half for STBC MIMOOFDM system as shown in Fig.5.

From the above reason, the PAPR reduction can employ only for the odd symbol sequence in the proposed method. Fig. 5 shows the proposed modified-PTS with concurrent algorithm for STBC MIMO-OFDM with two transmitted antennas. The PAPR reduction of two data symbol encoded by STBC as given in Eq. (2) will be operated only for the odd symbol sequence signal which can obtain the desired weighting factor for giving the optimum PAPR values. Meanwhile, the desired weighting factor of the even symbol sequence signal can obtain by mapping with the weighting factor of the odd symbol sequence signal which can be described in Table 1.

From Fig.5 and Table 1, it can be seen that the proposed modified-PTS with concurrent algorithm can reduce the PAPR with decreasing the SI-size for STBC MIMO-OFDM system.

\subsection{STBC decoder of information data for STBC MIMO-OFDM system with the proposed PAPR reduction method}

From Fig.1 and Eq.(1), the received signal at $R_{1}$ after FFT process can be given by the following equation
Table 1. Weighting factor mapping

\begin{tabular}{|c|c|c|c|c|}
\hline \multirow{2}{*}{ Symbols } & \multicolumn{3}{|c|}{ Cluster } \\
\cline { 2 - 4 } & \multicolumn{2}{|c|}{ 1st Part } & \multicolumn{2}{|c|}{ 2nd Part } \\
\hline \multirow{4}{*}{$\begin{array}{c}\text { Phase } \\
\text { Coefficient }\end{array}$} & \multicolumn{2}{|c|}{$\emptyset_{m}^{\text {part1,v }}$} & \multicolumn{2}{|c|}{$\emptyset_{m}^{\text {part2,v }}$} \\
\cline { 2 - 3 } & 0 & 0 & \multirow{2}{*}{} \\
\cline { 2 - 3 } & $\pi / 2$ & $3 \pi / 2$ & \multirow{2}{*}{ Eq.(9) } \\
\cline { 2 - 3 } & $\pi$ & $\pi$ & \multirow{2}{*}{} \\
\cline { 2 - 3 } & $3 \pi / 2$ & $\pi / 2$ & \multicolumn{2}{|c}{} \\
\hline
\end{tabular}

$$
\begin{gathered}
R_{m, n}=X_{m, n} H_{1, m, n}+X_{m+1, n} H_{2, m, n}, \\
R_{m+1, n}=-X_{m+1, n}^{*} H_{1, m+1, n}+X_{m, n}^{*} H_{2, m+1, n},
\end{gathered}
$$

where $H_{1}$ and $H_{2}$ are the channel frequency response between $T_{1}$ and $R_{1}$, and $T_{2}$ and $R_{1}$ respectively. In the assumption, the perfect channels $H_{1}$ and $H_{2}$ known at the receiver are employed to the data demodulation.

From Eq.(10), the decoded data signal $X_{m, n}$ and $X_{m+1, n}$ can be rewritten by

$$
\begin{gathered}
X_{m, n}=R_{m, n} H_{1, m, n}^{*}+R_{m+1, n}^{*} H_{2, m+1, n}, \\
X_{m+1, n}=-R_{m+1, n}^{*} H_{1, m+1, n}+R_{m, n} H_{2, m, n}^{*} .
\end{gathered}
$$

From Eqs.(12) and (13), the information data signal $\tilde{X}_{m, n}$ for odd symbol and $\tilde{X}_{m+1, n}$ for even symbol can be decoded correctly before demodulation by the following Eq. (14). 


$$
\begin{gathered}
\tilde{X}_{m, n}=\frac{X_{m, n}\left\{\left|H_{1, m+1, n}\right|^{2}+\left|H_{2, m, n}\right|^{2}\right\}-X_{m+1, n}\left\{H_{1, m, n}^{*} H_{2, m, n}-H_{1, m+1, n}^{*} H_{2, m+1, n}\right\}}{\left\{\left|H_{1, m, n}\right|^{2}+\left|H_{2, m+1, n}\right|^{2}\right\}\left\{\left|H_{1, m+1, n}\right|^{2}+\left|H_{2, m, n}\right|^{2}\right\}-\left\{H_{1, m, n}^{*} H_{2, m, n}-H_{1, m+1, n}^{*} H_{2, m+1, n}\right\}\left\{H_{1, m, n} H_{2, m, n}^{*}-H_{1, m+1, n} H_{2, m+1, n}^{*}\right\}}, \\
\tilde{X}_{m+1, n}=\frac{X_{m+1, n}\left\{\left|H_{1, m, n}\right|^{2}+\left|H_{2, m+1, n}\right|^{2}\right\}-X_{m, n}\left\{H_{1, m, n} H_{2, m, n}^{*}-H_{1, m+1, n} H_{2, m+1, n}^{*}\right\}}{\left\{\left|H_{1, m, n}\right|^{2}+\left|H_{2, m+1, n}\right|^{2}\right\}\left\{\left|H_{1, m+1, n}\right|^{2}+\left|H_{2, m, n}\right|^{2}\right\}-\left\{H_{1, m, n}^{*} H_{2, m, n}-H_{1, m+1, n}^{*} H_{2, m+1, n}\right\}\left\{H_{1, m, n} H_{2, m, n}^{*}-H_{1, m+1, n} H_{2, m+1, n}^{*}\right\}}
\end{gathered}
$$

\section{Simulation and discussion}

In this section, the parameters listed in Table 2 were used for evaluating by the computer simulation which the various results can demonstrate the effectiveness of the proposed method.

To get better PAPR for the proposed method by considering the optimum $\lambda$ value given in Eq.(9), the average PAPR value of the proposed method with $S=256$ patterns (maximum of possible number) for STBC MIMO-OFDM systems with two transmitted antennas is evaluated when changing $\lambda$ value as shown in Fig.6. In the figure, it can be seen that the optimum $\lambda$ value is taken by " 0 " because its average PAPR shows the lowest PAPR value even when increasing $M$ data subcarriers. From this result, it can be concluded that the PAPR optimization is not necessary to be employed in the 2nd part which leads to the reducing computational complexity of PAPR reduction.

Furthermore, the complexity in PAPR reduction methods can be calculated by

$$
\begin{aligned}
& \text { Conv.PTS }_{\text {Complexity }}=\frac{M}{V} \cdot W^{V} \\
& \text { Proposed }_{\text {Complexity }}=(1+\lambda) \frac{M \cdot S}{2 V}
\end{aligned}
$$

From Eq.(15), it can be seen that the computational complexity of the proposed PAPR reduction method can be considered by deciding $S$ phase pattern numbers. From this reason, the average PAPR is evaluated when changing $S$ phase pattern numbers as shown in Fig.7. In the Fig.7, it can be seen that the average PAPR values from $S=98$ to 256 patterns are small change. From this result, the optimum $S$ phase patterns in the proposed method are taken by " 98 " which is enough to get the lower PAPR for STBC MIMO-OFDM system. From the results in Figs. 6 and 7, the optimum $\lambda=0$ and $S=98$ patterns are taken in the proposed modified-PTS with ABC-Concurrent algorithm.

The CCDF function is evaluated as PAPR performance for the proposed PAPR reduction method which is shown in Fig.8. In the figure, the original STBC MIMO-OFDM [15] and the conventional PTS (conv.PTS) [14] methods are shown to compare with the proposed method. From the Fig.8, it can be seen that the proposed modifiedPTS with ABC-Concurrent algorithm shows the better PAPR which is approximately $2.4 \mathrm{~dB}$ and $0.2 \mathrm{~dB}$ gain over the original STBC MIMO-OFDM [15] and the conv.PTS [14] methods respectively. Unquestionably, the PAPR at $10^{-1}$ of CCDF dominates the degradation of BER in the non-linear

Table 2. Simulation parameters

\begin{tabular}{|l|c|}
\hline Information & Parameter \\
\hline Modulation technique & Coherent \\
\hline Demodulation & $5 \mathrm{MHz}$ \\
\hline OFDM occupied bandwidth & 256 \\
\hline$N$-points $I F F T / F F T$ & 64 \\
\hline$M$-data subcarriers & 4 and 4 \\
\hline$V$-clusters and $W$-weighting factor & Interleave \\
\hline Partition Type & $14.3 \mu \mathrm{S}$ \\
\hline Symbol duration & $1.3 \mu \mathrm{S}$ \\
\hline Duration of Guard interval $(\mathrm{GI})$ & 2 \\
\hline$r$-parameter of AM/AM-SSPA & $2 \times 1$ \\
\hline$T \times R$ antennas & STBC \\
\hline Encoder/Decoder & Exponential \\
\hline Multipath fading model & 9 and $1 \mathrm{~dB}$ \\
\hline Power delay profile & \multicolumn{2}{|l}{} \\
\hline Delay path and decay constant & \\
\hline
\end{tabular}

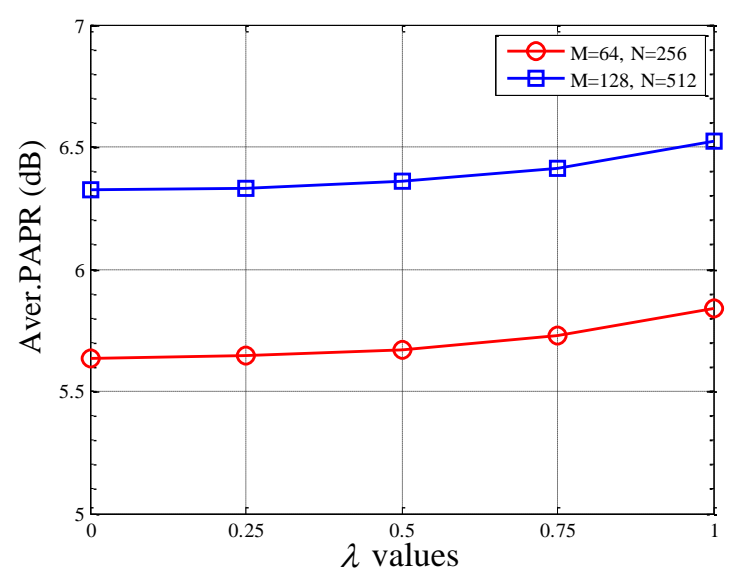

Figure.6 Average of PAPR vs. $\lambda$ values 
Table 3. Computational complexity between proposed and conventional PAPR reduction methods

\begin{tabular}{|c|c|c|c|}
\hline PAPR Reduction Methods & Complexity computed in Eq.(15) & SI-size \\
\hline Conventional PTS [14] & 4,096 & $100 \%$ & $T$ \\
\hline $\begin{array}{c}\text { Proposed modified-PTS with ABC-Concurrent } \\
\text { algorithm (S=98 patterns) }\end{array}$ & 784 & $\approx 19 \%$ & $T / 2$ \\
\hline
\end{tabular}

* $T$ is the number of transmit antennas

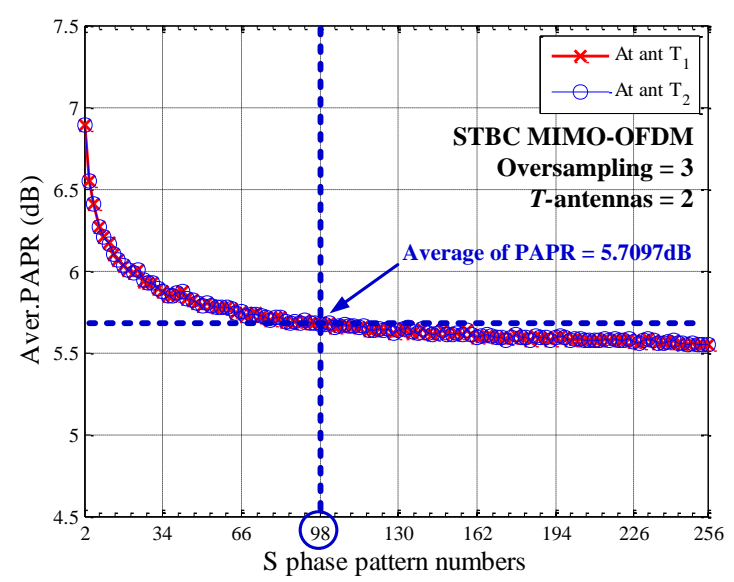

Figure.7 Average of PAPR vs. $S$ patterns

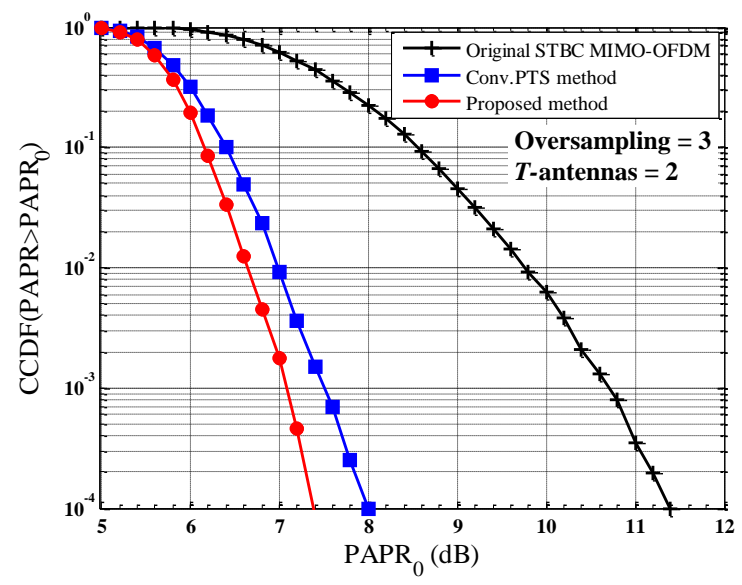

Figure. 8 Prob.(PAPR $>$ PAPR $\left._{0}\right)$ vs. PAPR $_{0}(\mathrm{~dB})$

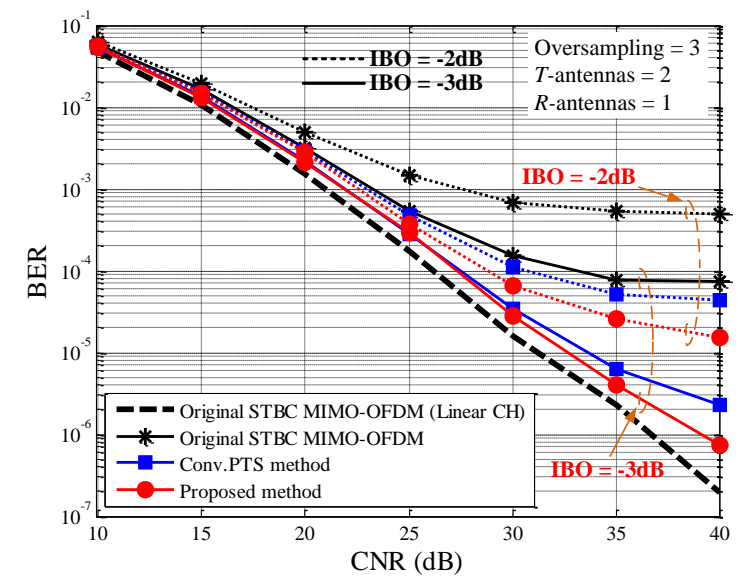

Figure.9 BER vs. CNR(dB)

channel. From this reason, it can be expected that the BER of STBC MIMO-OFDM systems with the
PAPR reduction by using the proposed modifiedPTS with ABC-Concurrent algorithm would be better than that with the PAPR reduction by using the conv.PTS method [14].

Fig. 9 shows the BER for the original STBC MIMO-OFDM and STBC MIMO-OFDM with PAPR reduction methods in the non-linear channel when changing the carrier-to-noise power ratio (CNR). From the figure, it can be seen that the proposed method can perform the better BER than those for the original STBC MIMO-OFDM [15] and the conv.PTS [14] methods respectively.

From Table 3 and the results in Figs 8 and 9, it can be concluded that the proposed modified-PTS with ABC-Concurrent algorithm can provide better both the PAPR and BER with low complexity than the conv.PTS method [14].

\section{Conclusions}

In this paper, the PAPR reduction by using the modified-PTS with ABC-Concurrent algorithm is proposed for STBC MIMO-OFDM systems in the non-linear channel. The silent feature of the proposed PAPR reduction method is to reduce the PAPR with decreasing the SI-size and reducing the complexity of PAPR reduction process by applying the proposed modified-PTS with ABC-Concurrent algorithm for STBC MIMO-OFDM system. The various computer simulation results show that the proposed modified-PTS with ABC-Concurrent algorithm can reduce PAPR by approximately 3.4 and $0.4 \mathrm{~dB}$ at $\mathrm{CCDF} 10^{-3}$ as comparing with the original STBC MIMO-OFDM and conventional PTS methods respectively, and can use the complexity approximately only $19 \%$ in PAPR reduction process which is decreased a lot from using the conventional PTS method even when increasing the number of transmitted antennas. Moreover, the proposed method can perform the better BER in the non-linear channel than the original STBC MIMO-OFDM and the conventional.PTS methods which is close to that of operating in non-linear channel.

\section{References}

[1] P.Aggarwall and V.A.Bohara, "A Nonlinear Downlink Multiuser MIMO-OFDM Systems", 
IEEE Wireless Communications Letters, Vol.6, No.3, pp.414-417, 2017.

[2] A.J. Al-Askery, C.C. Tsimenidis, S. Boussakta and J.A. Chambers, "Performance Analysis of Coded Massive MIMO-OFDM Systems Using Effective Matrix Inversion", IEEE Transactions on Communications, Vol.65, No.12, pp.52445256, 2017.

[3] S.M. Alamouti, "A simple transmit diversity technique for wireless communications", IEEE Journal on Selected Areas in Communications, Vol.16, No.8, pp.1451-1458, 1998.

[4] J.W. Jung, B.C. Kwon, H.W. Park, and J.S. Lim, "Superposition-based adaptive modulated space time block coding for MIMO-OFDM systems", IEEE Communications Letters, Vol.14, No.1, pp.30-32, 2010.

[5] S. Sengupta and B. K. Lande, "PAPR reduction in OFDM using Goppa codes", In: Proc. of International WIE Conf. on Electrical and Computer Engineering, Pune, India, pp.104-107, 2016.

[6] S. Jose and V. Kiran, "Adjecent partitioning PTS with TURBO coding for PAPR reduction in OFDM", In: Proc. of International Conf. on Advanced Computing and Communication Systems, pp.1-4, 2017.

[7] K. Anoh, C. Tanriover, B. Adebisi, and M. Hammoudeh, "A New Approach to Iterative Clipping and Filtering PAPR Reduction Scheme for OFDM Systems", IEEE Access, Vol.6, pp.17533-17544, 2018.

[8] M. Mounir, M.I. Youssef, and I.F. Tarrad, "On the effectiveness of deliberate clipping PAPR reduction technique in OFDM systems", In: Proc. of International Japan-Africa Conf. on Electronics, Communications and Computers, pp.21-24, 2017.

[9] D.J.G. Mestdagh, J.L.G. Monsalve, and J.M. Brossier, "Green OFDM: a new selected mapping method for OFDM PAPR reduction", IEEE Electronics Letters, Vol.54, No.7, pp.449450, 2018.

[10] H.Y. Liang, K.C. Chou, and H.C. Chu, "A modified SLM scheme with two-stage scrambling for PAPR reduction in OFDM systems", In: Proc. of International Conf. on Awareness Science and Technology, pp.215-218, 2017.

[11] X. Cheng, D. Liu, S. Feng, Q. Pan, and H.F. Fang, "PTS based on DisABC algorithm for PAPR reduction in OFDM systems", IEEE Electronics Letters, Vol.54, No.6, pp.397-398, 2018.
[12] H.S. Chen and K.C. Chung, "A PTS Technique With Non-Disjoint Sub-Block Partitions in $\mathrm{M}$ QAM OFDM Systems", IEEE Transactions on Broadcasting, Vol.64, No.1, pp.146-152, 2018.

[13] C. Dudak, A.T. Koc, and S. Koc, "Solid state power amplifier (SSPA) nonlinearity effects on quadri-phase shift keying modulation", In: Proc. of International Conf. on Wireless Technology, pp.237-240, 2004.

[14] P. Boonsrimuang, K. Mori, T. Paungma, and H. Kobayashi, "Proposal of improved PTS method for OFDM signal", In: Proc. of International Conf. Symposium on Personal, Indoor and Mobile Radio Communications, pp.1-5, 2007.

[15] T. Mata, P. Boonsrimuang, P. Boonsrimuang, and H. Kobayashi, "Proposal of improved PTS method for STBC MIMO-OFDM system", IEICE Transactions on Communications, Vol. E93-B, pp. 2673-2676, 2010.

[16] P. Jirajaracheep, T. Mata, and P. Boonsrimuang, "A PAPR Reduction in FBMC-OQAM System via Overlapping-PTS with Artificial Bee Colony Phase Optimization", International Journal of Intelligent Engineering and System, Vol. 12, No. 5, pp. 249-256, 2019. 